\title{
Automatic Classification of Small Bowel Mucosa Alterations in Celiac Disease for Confocal Laser Endomicroscopy
}

\author{
Davide Boschetto $^{\mathrm{a}, \mathrm{b}}$, Gianluca Di Claudio ${ }^{\mathrm{b}}$, Hadis Mirzaei ${ }^{\mathrm{c}}$, Rupert Leong ${ }^{\mathrm{c}}$, and Enrico Grisan ${ }^{\mathrm{b}}$ \\ ${ }^{a}$ IMT School for Advanced Studies Lucca, Lucca, Italy \\ ${ }^{\mathrm{b}}$ Department of Information Engineering, Universitá degli Studi di Padova, Padova, Italy \\ ${ }^{\mathrm{c}}$ Gastroenterology and Liver Services, Sydney South West Area Health Service, Bankstown \\ Hospital, Faculty of Medicine, The University of New South Wales, Sydney, Australia
}

\begin{abstract}
Celiac disease $(\mathrm{CD})$ is an immune-mediated enteropathy triggered by exposure to gluten and similar proteins, affecting genetically susceptible persons, increasing their risk of different complications. Small bowels mucosa damage due to CD involves various degrees of endoscopically relevant lesions, which are not easily recognized: their overall sensitivity and positive predictive values are poor even when zoom-endoscopy is used. Confocal Laser Endomicroscopy (CLE) allows skilled and trained experts to qualitative evaluate mucosa alteration such as a decrease in goblet cells density, presence of villous atrophy or crypt hypertrophy. We present a method for automatically classifying CLE images into three different classes: normal regions, villous atrophy and crypt hypertrophy. This classification is performed after a features selection process, in which four features are extracted from each image, through the application of homomorphic filtering and border identification through Canny and Sobel operators. Three different classifiers have been tested on a dataset of 67 different images labeled by experts in three classes (normal, VA and $\mathrm{CH}$ ): linear approach, Nave-Bayes quadratic approach and a standard quadratic analysis, all validated with a ten-fold cross validation. Linear classification achieves $82.09 \%$ accuracy (class accuracies: $90.32 \%$ for normal villi, $82.35 \%$ for VA and $68.42 \%$ for $\mathrm{CH}$, sensitivity: 0.68 , specificity 1.00 ), Nave Bayes analysis returns $83.58 \%$ accuracy $(90.32 \%$ for normal villi, $70.59 \%$ for VA and $84.21 \%$ for $\mathrm{CH}$, sensitivity: 0.84 specificity: 0.92$)$, while the quadratic analysis achieves a final accuracy of $94.03 \%(96.77 \%$ accuracy for normal villi, $94.12 \%$ for VA and $89.47 \%$ for $\mathrm{CH}$, sensitivity: 0.89 , specificity: 0.98 ).
\end{abstract}

\section{INTRODUCTION}

Celiac disease (CD) is one of the most frequent immune-mediated enteropathy that affects genetically susceptible persons triggered by exposure to gluten and similar proteins. This disease is a hidden epidemic: most of the celiac patients will in fact remain undiagnosed during their life. Exposure to gluten causes variable damage to the small bowel mucosa: mild damage include cases with increased number of intraepithelial lymphocytes and the presence of Crypt Hyperplasia $(\mathrm{CH})$, while severe forms of the lesions involve various degrees of endoscopically relevant lesions such as villous Atrophy (VA). ${ }^{1}$ Overall sensitivity and positive predictive values of VA and $\mathrm{CH}$ are poor even when zoom endoscopy is used, ${ }^{2}$ implying that these two alterations of the mucosa are not easily recognized during endoscopy. Thus, in everyday practice, the identification of CD is made on the basis of a positive diagnostic intestinal biopsy and of the concomitant presence of a positive celiac serology. ${ }^{3}$ The gold standard in the diagnosis of $\mathrm{CD}$ is the demonstration of VA in duodenal biopsies. ${ }^{4}$ VA is investigated extensively in the medical community, ${ }^{5-7}$ and image processing methods as well as quantitative computational methods are highly needed, required and recommended from the community for the characterization of the small intestinal mucosa in suspected and known CD patients. ${ }^{8}$ At variance with zoom endoscopy, confocal laser endomicroscopy (CLE) is a relatively novel endoscopic method that permits on-site microscopy of the gastrointestinal mucosa after the application of a fluorescent agent, allowing the experienced endoscopists to diagnose VA, $\mathrm{CH}$ as well as intraepithelial lymphocytes with high accuracy. ${ }^{9}$ Images originating from CLE, as Fig. 1 shows, are very informative about the status of small bowel mucosa: villi, crypts and goblet cells can be clearly discerned,

Further author information: (Send correspondence to Davide Boschetto)

Davide Boschetto: E-mail: davide.boschetto@imtlucca.it 

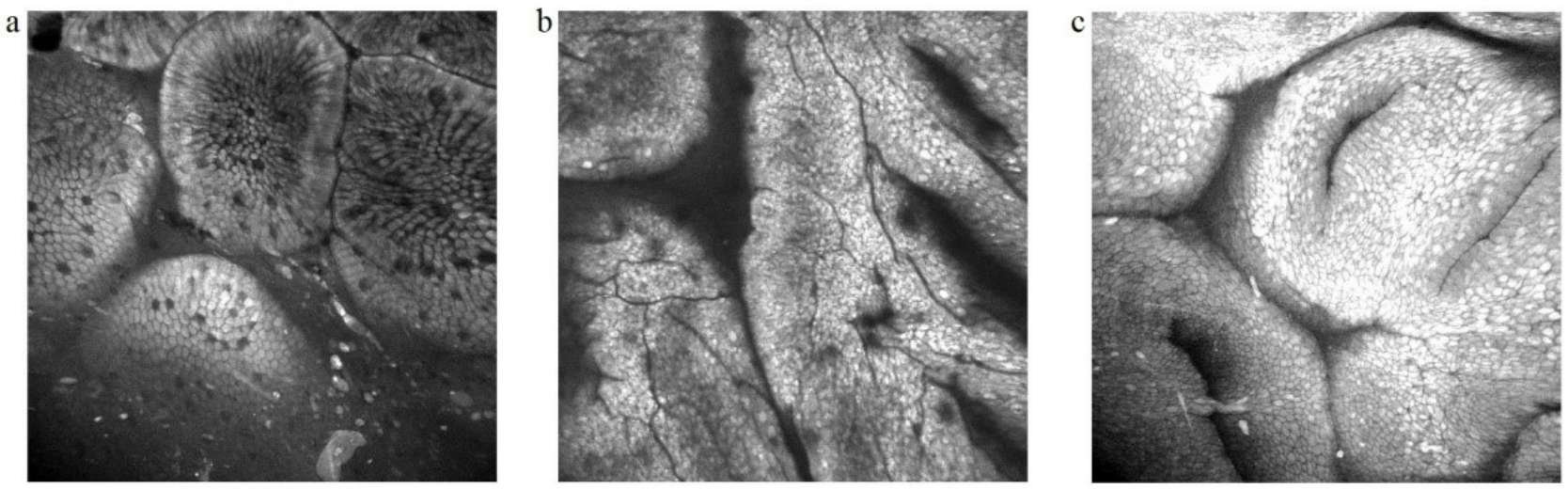

Figure 1. Three images from the dataset, one for each class: (a) normal epithelium, with visible goblet cells; (b) villous atrophy; (c) villous atrophy with crypt hypertrophy.

depending on the region of the mucosa under analysis and the staging of the patient. Villous atrophy, along with crypt hyperplasia and goblet cells density in the duodenal epithelium, is an important marker of mucosa damage, ${ }^{10-12}$ and a useful factor for staging CD severity: hence, an automatic tool able to differentiate features and distinguish images based on those features would be very important for the process of staging and grading the severity of the disease. This work proposes four simple features, extracts them, compares the performances of three different classifiers and serves as a first approach for estimating the difficulties that needs to be overcame to achieve satisfying automatic classification of confocal endomicroscopy images.

To our knowledge, no previous work has been published on image processing methods with the purpose of automatically classify and stage celiac disease from images of confocal endomicroscopy. This is not a trivial process: villi are highly textured and present high variability in appearance, shape and dimension. In CLE images, they can exhibit smooth and fuzzy borders among (and between) villi and inter-villous space. Among villi, vessels can be found in inter-villous space, with goblet cells. In severe CD stages, a possible collapse of all villi into a uniform mucosa that is depleted of villi can be observed. Other than this, possible presence of crypts and fluorescine leakage can prevent accurate detection with standard image processing methods.

This work's main purpose (to be inserted in our ongoing development effort of developing a CADSS for CLE studies $^{13,14}$ ) is to develop an automatic pipeline to classify images from CLE studies, since images acquired from patients suffering from mucosa alteration such as villous atrophy or crypt hypertrophy are sensitively different from ones in the normal situation, as shown in Fig. 1.

Normally (Fig. 1, left), villi are distinguishable one from the other. If Villous Atrophy is present (Fig. 1, center), the mucosa is flattened, resulting in villi disappearing in the focal plane. When Crypt Hypertrophy is present, cells volume increase, resulting in bridges among different villi and more visible crypts (Fig. 1, right). Automatic grading of such problems could potentially result in in-vivo virtual biopsies or in targeted biopsies (instead of using the four-quadrant protocol), decreasing the need for potentially dangerous multiple biopsies to grade the disease.

\section{MATERIALS}

In this study, 67 images (class distribution: 31 normal images, 17 with VA and 19 with $\mathrm{CH}$ ) confocal images were obtained from clinical examinations conducted at the Gastroenterology and Liver Services of the BankstownLidcombe Hospital (Sydney, Australia). ${ }^{15}$ Each patient underwent a confocal gastroscopy (Pentax EC-3870FK, Pentax, Tokyo, Japan) under conscious sedation and with a IV aliquots of fluorescein sodium and topical acriflavine hydrocloride to enhance images. Each image represent a mucosal region of $0.5 \times 0.5 \mathrm{~mm}$, with an in-plane resolution of $2 \mathrm{pixel} / \mu \mathrm{m}$, resulting in images of $1024 \times 1024$ pixels. Each image was labeled by an expert as Normal, VA or CH. All the images used in this work show a mucosal region entirely filled by villous folds, with negligible inter-villous space. 

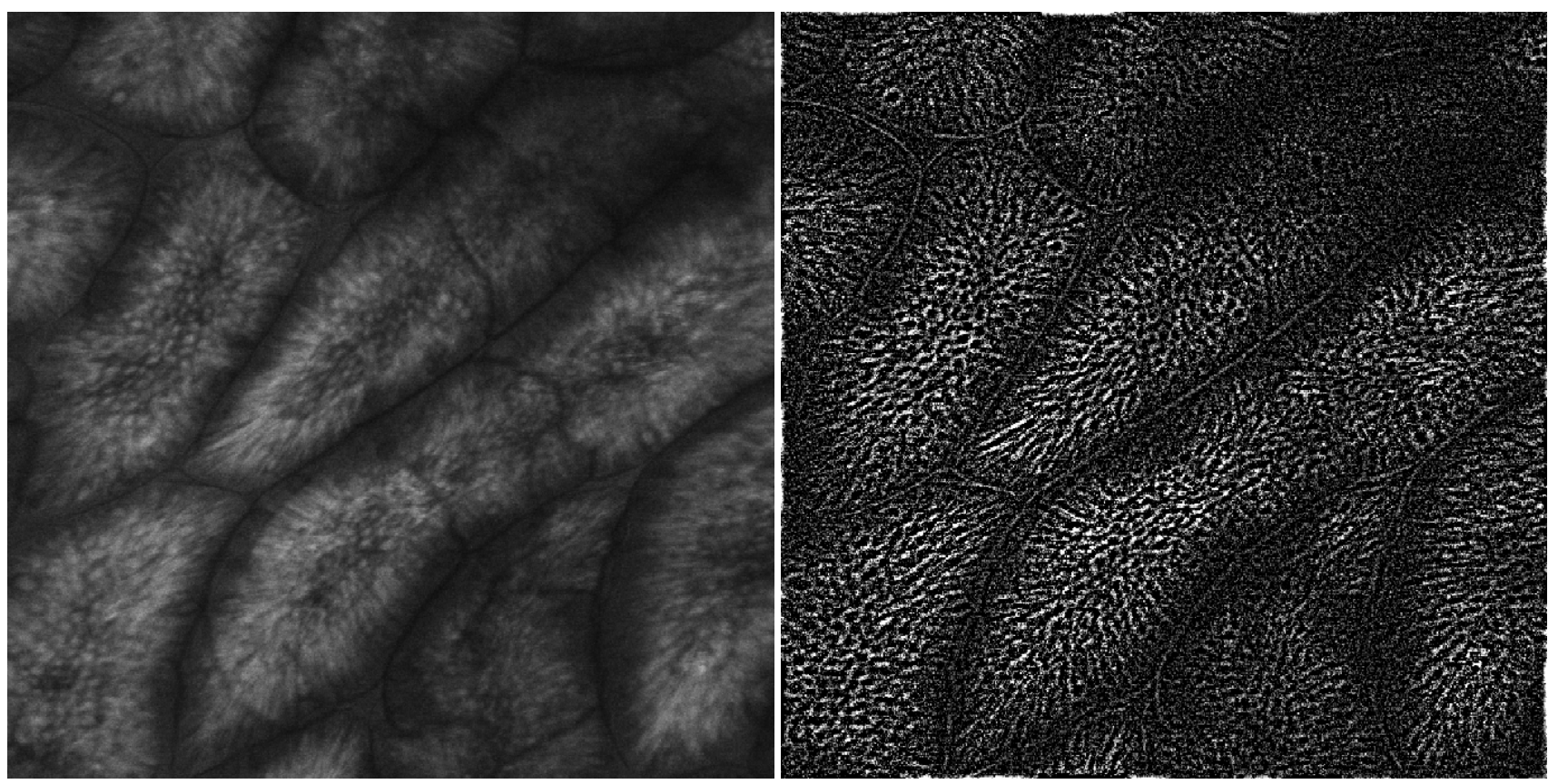

Figure 2. Original image from the dataset (left) and processed image through homomorphic filtering (right), with greyscale negavite rescaled intensities for visualization purposes.

\section{METHODS}

The first part of the work is based on the extraction of four features. Two features are extracted from images processed via edge-based filtering, while the remaining two depends from homomorphic filtering.

\subsection{Homomorphic Filtering}

Every image can be represented, using a multiplicative noise model, as a 2D function:

$$
I(x, y)=L(x, y) R(x, y),
$$

where the first term represents the illumination (low frequencies) and the latter represents the reflectance (high frequencies). Homomorphic filtering is a technique ${ }^{16,17}$ that can remove multiplicative noise responding to certain characteristics. Transposing the above model in the logarithmic domain, the multiplicative components are represented as additive components. Then, a high-pass filter needs to be applied to decrease the low-pass illumination component and enhance the reflectance high-frequency component. To do this, a DFT is applied to each image, to operate in the frequency domain. A high-pass filter can be obtained starting from a Gaussian filter, a Butterworth filter or a Chebychev filter. We chose to use a modified Butterworth filter, given its property of soft slope in the cutoff frequency. Butterworth high-pass filter has a transfer function $H(u, v)$ defined as

$$
H_{B}(u, v)=\frac{1}{1+\left(\frac{D_{0}}{D(u, v)}\right)^{2 n}}
$$

where $D_{0}$ is the cutoff frequency, $D(u, v)$ represents the distance from the center and $n$ is its order. If we edit the numerator to take into account the low- and high-frequency gain $\left(\gamma_{L}\right.$ e $\gamma_{H}$ respectively), we obtain a homomorphic filter as

$$
H_{H}(u, v)=\frac{\gamma_{H}-\gamma_{L}}{1+\left(\frac{D_{0}}{D(u, v)}\right)^{2 n}} .
$$

After applying this filter, the resulting image is anti-transformed into spatial coordinates and from log-space back into its original domain, obtaining the image $I_{b}(x, y)$. At this point, all the average greyscale intensity values $i_{h}$, 

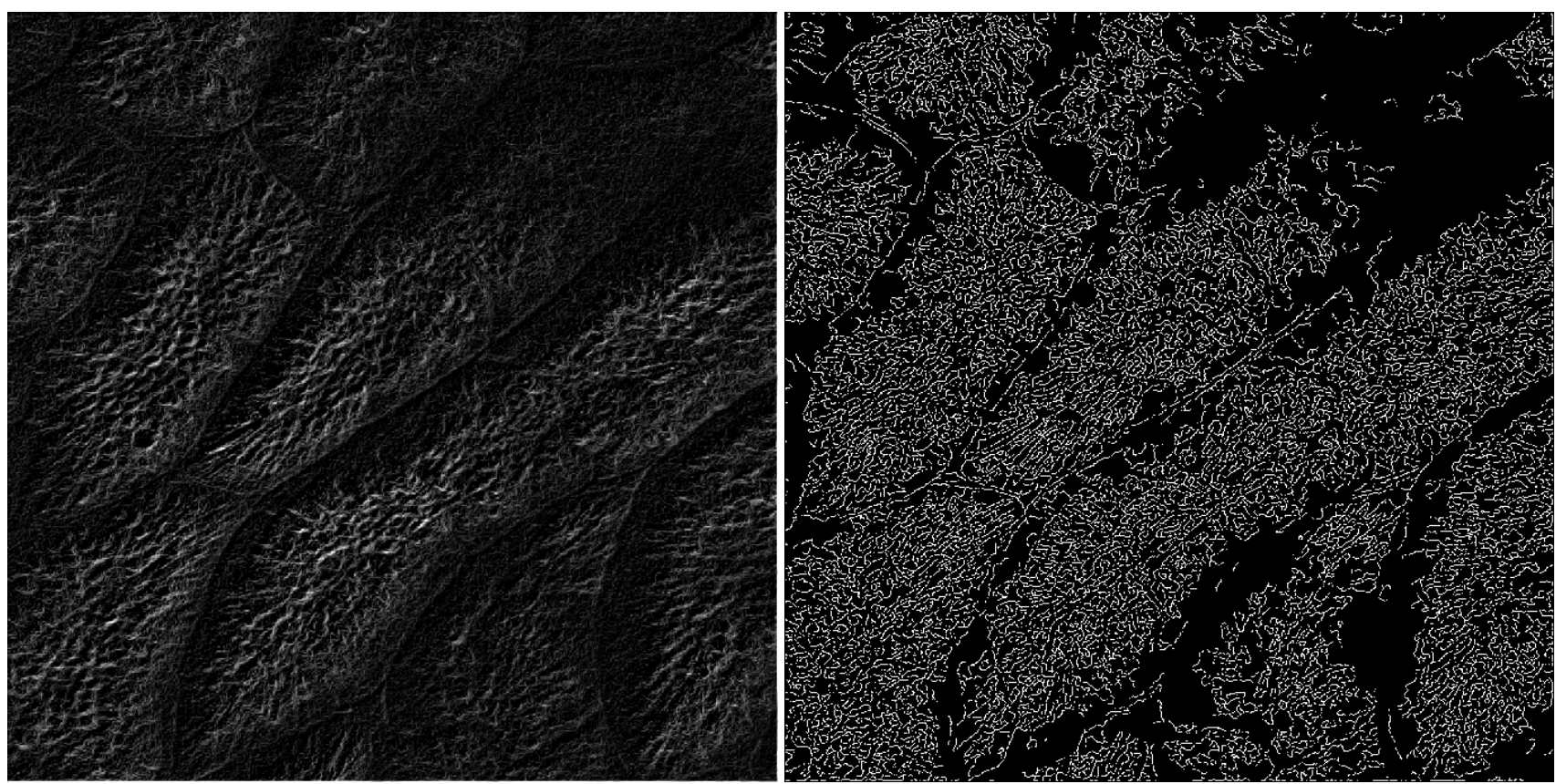

Figure 3. Sobel (left) and Canny (right) edge detection methods applied to the same image from the dataset as in Fig. 2.

one for each image in the dataset, were stored as first feature. An example of an image from the dataset with homomorphic filter applied, is shown in Fig. 2.

A second image, $I_{e}$, has been computed as such:

$$
I_{e}=I+I_{h},
$$

by computing the pixel-wise sum of the original image with $I_{h}$. The second feature is the average intensity of such image, $i_{e}$. An example of this is shown in Fig. 2.

\subsection{Edge-based features}

Two operators widely used in image processing for edge detection algorithms are the Sobel-Feldman operator and the Canny operator. The Sobel operator is a discrete differentiation operator that approximates the gradient of the image intensity function through convolution of two predetermined $3 \times 3$ masks. Canny edge detection, instead, calculates the gradient using the derivative of a Gaussian filter, using two thresholds to detect local strong and weak edges, being therefore less affected by noise, and its output is a binary image. The two features extracted from this pipeline are the mean intensities of the Sobel approximation of the gradient and the mean intensity obtained from Cannys edge approximation binary image. An example of these two images is shown in Fig. 3.

\subsection{Classification}

The four features are used to train three different classifiers. Under the hypothesis of multivariate normal distribution, the linear approach models each class with the same covariance matrix, while the mean varies in each class. For both quadratic approach, both mean and covariance are considered varying in each class. Classification is achieved in by inferring mean and covariance parameters of each class. As first step, the sample mean is computed for each class. Then, the sample covariance is computed by subtracting the sample mean of each class from all the samples belonging to that class, taking the empirical covariance matrix of each class (or of all the classes, in the linear case). The difference between the two quadratic classifiers is how the covariance matrix is treated: Nave Bayes approach uses only its diagonal, to account for possible singular covariance matrices, using the pseudo-inverse if necessary. This, because it is assumed that all variables are conditionally 
independent given the class label (therefore the name Nave Bayes). Standard quadratic classification instead uses the full covariance matrix, hence being available only with non-singular covariance matrices. All classifications are validated using a standard 10 -fold cross validation scheme. Total and per-class accuracy are computed, along with sensitivity and specificity for each case.

\section{RESULTS}

A total of 67 images (class distribution: 31 normal images, 17 with VA and 19 with $\mathrm{CH}$ ) have been processed and classified with the algorithms described above. All images were obtained from previous clinical trials conducted at the Gastroenterology and Liver Services of the Bankstown-Lidcombe Hospital (Sydney, Australia). The implementation has been carried out using MATLAB R2015a. Accuracies (overall and for each class), sensitivities and specificities are shown in Table 1 and the three confusion matrices are shown in Fig. 4.

\begin{tabular}{c|c|c|c|c|c|c} 
& Accuracy* & Acc-Normal & Acc-VA* & Acc-CH* & TPR & TNR \\
Linear & 82.09 & 90.32 & 82.35 & 68.42 & 0.68 & 0.98 \\
Nave Bayes Quadratic & 83.58 & 87.10 & 76.47 & 84.21 & 0.84 & 0.94 \\
Quadratic & 94.03 & 96.77 & 94.12 & 89.47 & 0.89 & 0.98
\end{tabular}

Table 1. Total and per-class accuracies, sensitivity and specificity for each classification complexity. *Accuracies are reported as percentages, while TPR and TNR are reported on 0-1 scale.

We can note how overall results reflect the complexity of the classification analysis. Generally, all classifiers show good performances, with the standard quadratic obtaining the best scores in all situations. Although, it can be noted that linear classification is more accurate in detecting both normal and Villous Atrophy images if compared with quadratic Nave Bayes. The lower overall accuracy of the linear classifier is due to inaccuracies in the classification of images labeled as $\mathrm{CH}$, implying the need for a quadratic formulation to better cluster images belonging to that, and other, classes.
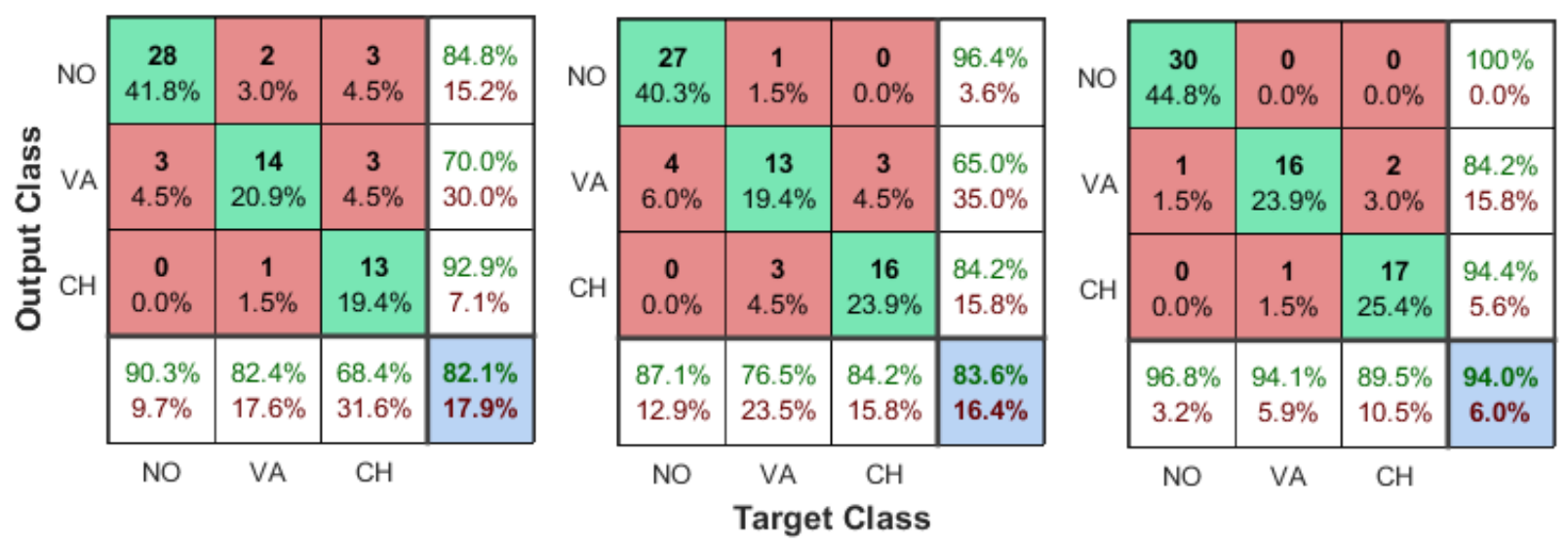

Figure 4. Confusion matrices for three-class comparison (Normal, Villous Atrophy and Crypt Hypertrophy for three different classifiers: (Left) Linear (Middle) Nave Bayes (Bottom) Quadratic. Overall accuracies and error can be found in each bottom-right cell, while overall accuracies are found in the bottom row of each sub-figure.

\section{CONCLUSIONS}

Our method, albeit simple, proves to be effective for the detection and classification of confocal endomicroscopy images. The main drawback of the method is that it requires a presence of villous folds in the full field of view, since it extracts global features. As future works, our group is focusing on editing this algorithm to work locally instead of globally: this will improve the reliability of our tool in case of images with partial occlusions, variable depth or mucus in the field of view. In our ongoing development of a CADSS for the purpose of Celiac Disease staging from Confocal Laser Endomicroscopy images, this work achieves good results with solid, widely used and robust image processing techniques. 


\section{REFERENCES}

[1] Mulder, C., van Weyenberg, S., and Jacobs, M., "Celiac disease is not yet mainstream in endoscopy," Endoscopy 42(3), 218-9 (2010).

[2] Dewar, D. and Ciclitira, P., "Clinical features and diagnosis of celiac disease," Gastroenterology 128(Suppl1), S19-S24 (2005).

[3] Fasano, A. and Catassi, C., "Current approaches to diagnosis and treatment of celiac disease: an evolving spectrum," Gastroenterology, 120(3), 636-51 (2001).

[4] Ciaccio, E., Lewis, S., and Green, P., "Detection of villous atrophy using endoscopic images for the diagnosis of celiac disease," Digestive Diseases and Sciences 58(8), 1167-9 (2013).

[5] Bonamico, M., Mariani, P., Thanasi, E., Ferri, M., Nenna, R., Tiberti, C., Mora, B., Mazzilli, M., and Magliocca, F., "Patchy villous atrophy of the duodenum in childhood celiac disease," J Pediatr Gastroenterol Nutr 38(2), 204-7 (2004).

[6] Lundin, K., Nilsen, E., Scott, H., Løberg, E., Gjøen, A., Bratlie, J., Skar, V., Mendez, E., Løvik, A., and Kett, K., "Oats induced villous atrophy in coeliac disease," Gut 52(11), 1649-52 (2003).

[7] Hüe, S., Mention, J., Monteiro, R., Zhang, S., Cellier, C., Schmitz, J., Verkarre, V., Fodil, N., Bahram, S., Cerf-Bensussan, N., and Caillat-Zucman, S., "A direct role for NKG2D/MICA interaction in villous atrophy during celiac disease," Immunity 21(3), 367-77 (2004).

[8] Ciaccio, E., Bhagat, G., Lewis, S., and Green, P., "Quantitative image analysis of celiac disease," World $J$ Gastroenterol 21(9), 2577-81 (2015).

[9] Venkatesh, K., Abou-Taleb, A., Cohen, M., Evans, C., Thomas, S., Oliver, P., Taylor, C., and Thomson, M., "Role of confocal endomicroscopy in the diagnosis of celiac disease," J Pediatr Gastroenterol Nutr 51(3), 274-9 (2010).

[10] Brown, I., Whiteman, D., and Lauwers, G., "Foveolar type dysplasia in barrett esophagus," Modern Pathology 23, 834-43 (2010).

[11] Ali, N., "Understanding celiac disease: An introduction for patients and caregivers," Rowman and Littlefield Publishers (2014).

[12] Mirzaei, H., Fung, C., Chang, J., and Leong, R., "Confocal laser endomicroscopy is equivalent to histology in the detection of gluten-free diet efficacy in coeliac disease," Journal of Gastroenterology and Hepatology, Special Issue: Australian Gastroenterology Week 2014 Gold Coast Convention and Exhibition Centre, Broadbeach, Queensland, 22-24 October 201429(S2), 53 (2014).

[13] Boschetto, D., Mirzaei, H., Leong, R., Tarroni, G., and Grisan, E., "Semiautomatic detection of villi in confocal endoscopy for the evaluation of celiac disease," Conf Proc IEEE Eng Med Biol Soc. , 8143-8146 (2015).

[14] Boschetto, D., Mirzaei, H., Leong, R., and Grisan, E., "Detection and density estimation of goblet cells in confocal endoscopy for the evaluation of celiac disease," Conf Proc IEEE Eng Med Biol Soc. , 6248-6251 (2015).

[15] Leong, R., Nguyen, N., Meredith, C., Al-Sohaily, S., Kukic, D., Delaney, P., Murr, E., Yong, J., Merrett, N., and Biankin, A., "In vivo confocal endomicroscopy in the diagnosis and evaluation of celiac disease," Gastroenterology 135(6), 1870-6 (2008).

[16] Ponomarev, V. and Pogrebnyak, O., "Image enhancement by homomorphic filters," Applications of Digital Image Processing XVIII 2564, 153-159 (1995).

[17] Pitas, I. and Venetsanopoulos, A., "Homomorphic filters," in [Nonlinear Digital Filters], ch. 7, 217-243, Springer US (1990). 